\title{
Performance in mortality prediction of SAPS 3 And MPM-III scores among adult patients admitted to the ICU of a private tertiary referral hospital in Tanzania: A retrospective cohort study
}

\author{
Nadeem Kassam ${ }^{\text {Corresp., } 1}{ }^{,}$Eric Aghan ${ }^{2}$, Samina Somji ${ }^{1}$, Omar Aziz $^{3}$, James Orwa $^{4}$, Salim R Surani $^{5}$ \\ 1 Internal medicine, Aga Khan University, Dar-es-Salaam, Tanzania \\ ${ }^{2}$ Family medicine, Aga Khan University, Dar-es-Salaam, Tanzania \\ 3 Internal medicine, Aga Khan Hospital, Dar-es-Salaam, Tanzania \\ 4 Population health, Aga Khan University, Nairobi, Kenya \\ 5 Medicine \& Pharmacy, Texas A\&M University, Texas, United States \\ Corresponding Author: Nadeem Kassam \\ Email address: nadeem.kassam@aku.edu
}

Background: Illness predictive scoring systems are significant and meaningful adjuncts of patient management in the Intensive Care Unit (ICU). They assist in predicting patient outcomes, improve clinical decision making and provide insight into the effectiveness of care and management of patients while optimizing the use of hospital resources. We evaluated mortality predictive performance of Simplified Acute Physiology Score (SAPS 3) and Mortality Probability Models ( $\left.\mathrm{MPM}_{0}-\mathrm{III}\right)$ and compared their performance in predicting outcome as well as identifying disease pattern and factors associated with increased mortality. Methods: This was a retrospective cohort study of adult patients admitted to the ICU of the Aga Khan Hospital, Dar- es- Salaam, Tanzania between August 2018 and April 2020. Demographics, clinical characteristics, outcomes, source of admission, primary admission category, length of stay and the support provided with the worst physiological data within the first hour of ICU admission were extracted. SAPS 3 and MPM $_{0}$-III scores were calculated using an online web-based calculator. The performance of each model was assessed by discrimination and calibration. Discrimination between survivors and non survivors was assessed by the area under the receiver operator characteristic curve (ROC) and calibration was estimated using the Hosmer-Lemeshow goodness-of-fit test. Results: A total of 331 patients were enrolled in the study with a median age of 58 years (IQR 43-71), most of whom were male $(n=208,62.8 \%)$, of African origin $(n=178,53.8 \%)$ and admitted from the emergency department $(n=306,92.4 \%)$. In- hospital mortality of critically ill patients was $16.1 \%$. Discrimination was very good for all models, the area under the receiver-operating characteristic $(\mathrm{ROC})$ curve for SAPS 3 and $\mathrm{MPM}_{0}-\mathrm{III}$ was $0.89(95 \% \mathrm{Cl}$ : 
$0.844-0.935)$ and $0.90(95 \% \mathrm{Cl}: 0.864-0.944)$ respectively. Calibration as calculated by Hosmer-Lemeshow goodness-of-fit test showed good calibration for SAPS 3 and MPM $_{0}$-III with Chi- square values of 4.61 and 5.08 respectively and $P$ - Value $>0.05$. Conclusion: Both SAPS 3 and MPM $_{0}$-III performed well in our cohort of patients admitted to the intensive care unit of a private tertiary hospital. The in-hospital mortality of critically ill patients was lower compared to studies done in other intensive care units in tertiary referral hospitals within Tanzania. 
Primary Author: Nadeem Mehboob Kassam ${ }^{1}$

Co-Authors: Eric Luggar Aghan ${ }^{2}$, Samina Sadrudin Somji ${ }^{1}$, Omar Mohamed Aziz ${ }^{3}$, James Orwa Kenyatta ${ }^{4}$, Salim Ramzan Surani ${ }^{5}$

1. Department of Internal Medicine, Aga Khan University, Dar es Salaam, Tanzania

2. Department of Family Medicine, Aga Khan University, Dar es Salaam, Tanzania

3. Department of Internal Medicine, Aga Khan Hospital, Dar es Salaam, Tanzania

4. Department of Population Health, Aga Khan University, Nairobi, Kenya

5. Department of Medicine \& Pharmacy, Texas A\&M University, Texas, USA

Institutional address

1. Nadeem Kassam : Nadeem.kassam@aku.edu

2. Eric Aghan : eric.aghan@aku.edu

3. Samina Somji : samina.somji@aku.edu

4. Omar Aziz : omar.aziz@akhst.org

5. James Orwa : james.orwa@aku.edu

6. Salim Surani : srsurani@hotmail.com

Corresponding author: Nadeem Kassam

Nadeem.kassam@aku.edu 


\section{ABSTRACT}

\section{Background}

36 Illness predictive scoring systems are significant and meaningful adjuncts of patient management in the

37 Intensive Care Unit (ICU). They assist in predicting patient outcomes, improve clinical decision making 38 and provide insight into the effectiveness of care and management of patients while optimizing the use of 39 hospital resources. We evaluated the mortality predictive performance of the Simplified Acute Physiology 40 Score (SAPS 3) and Mortality Probability Models (MPM $\left.\mathrm{M}_{0}-\mathrm{III}\right)$ and compared their performance in 41 predicting outcomes as well as identifying disease patterns and factors associated with increased 42 mortality.

\section{Methods}

45 This was a retrospective cohort study of adult patients admitted to the ICU of the Aga Khan Hospital, Dar 46 - es Salaam, Tanzania between August 2018 and April 2020. Demographics, clinical characteristics, 47 outcomes, source of admission, primary admission category, length of stay and the support provided with 48 the worst physiological data within the first hour of ICU admission were extracted. SAPS 3 and $\mathrm{MPM}_{0}$-III 49 scores were calculated using an online web-based calculator. The performance of each model was 50 assessed by discrimination and calibration. Discrimination between survivors and non-survivors was 51 assessed by the area under the receiver operator characteristic curve (ROC) and calibration was estimated 52 using the Hosmer-Lemeshow goodness-of-fit test.

\section{Results}

54 A total of 331 patients were enrolled in the study with a median age of 58 years (IQR 43-71), most of 55 whom were male $(\mathrm{n}=208,62.8 \%)$, of African origin $(\mathrm{n}=178,53.8 \%)$ and admitted from the emergency 56 department ( $\mathrm{n}=306,92.4 \%$ ). In-hospital mortality of critically ill patients was $16.1 \%$. Discrimination was 57 very good for all models, the area under the ROC curve for SAPS 3 and $\mathrm{MPM}_{0}$-III was 0.89 (95\%CI: 58 0.844-0.935) and 0.90 (95\%CI: 0.864-0.944) respectively. Calibration as calculated by Hosmer59 Lemeshow goodness-of-fit test showed good calibration for SAPS 3 and MPM $_{0}$-III with chi-square values 60 of 4.61 and 5.08 respectively and P-Value $>0.05$.

61 Conclusion

62 Both SAPS 3 and MPM $_{0}$-III performed well in our cohort of patients admitted to the intensive care unit of 63 a private tertiary hospital. The in-hospital mortality of critically ill patients was lower compared to studies 64 done in other intensive care units in tertiary referral hospitals within Tanzania. 


\section{BACKGROUND}

67 The burden of critical care and ICU mortality is greatest in countries with low global national income (1).

68 The reported ICU mortality widely varies from one setting to the other with higher rates reported in low 69 and middle-income countries (LMICs) (1-3). As of $1^{\text {st } J u l y ~ 2020, ~ t h e ~ W o r l d ~ B a n k ~ u p g r a d e d ~ T a n z a n i a ' s ~}$ economic status from a low to lower-middle income country due to its strong economic performance over the past decade. However, the availability of intensive care units in Tanzania is very limited; none of the seven district hospitals surveyed in 2009 had an ICU. The four national referral hospitals had a total of only 38 ICU beds serving a population of 57 million (4). This is in contrast to high-income countries (HICs) which generally have between 5 to 30 ICU beds per 100000 people (5). The availability and improvement of quality of care of critical illness in LMICs is necessary to reduce this burden and even more significant in the coming years as the population ages and prevalence of comorbidities increases (6). Despite the use of high-cost and sophisticated devices, ICU mortality rates remain high. The burden of diseases compounded by a severe lack of resources, specialists and data makes prediction of ICU outcomes in terms of morbidity and mortality a crucial component of care across the continent. In HICs mortality prediction models are not only used to predict outcome but also as tools for quality enhancement and analytical decision making. These mortality predictive models were developed more than 25 years ago using patient characteristics. They help quantify the severity of illness, estimate the gravity of the disease, help predict outcome and assist in resource allocation $(7,8)$.

The three major predictive scoring systems used to predict mortality in general ICU patients are the Acute Physiologic and Chronic Health Evaluation (APACHE) scoring system, the Simplified Acute Physiologic Score (SAPS) and the Mortality Prediction Model $\left(\mathrm{MPM}_{0}\right)$ (9). APACHE-IV, SAPS 3, and MPM $\mathrm{M}_{0}$ III are the latest versions of the aforementioned scoring systems (10-12). When selecting a predictive scoring system for use in a given ICU, it is essential to use a model that is well-proven, established and validated contextually. APACHE-IV has long been considered more precise for predicting mortality but is perceived as burdensome and more costly especially in resource-limited settings (13). MPM $\mathrm{M}_{0}$-III is considered superior in resource-limited settings since it has the lowest extraction burden among the three models and is available without cost on various medical information sites. MPM$_{0}$-III has been a wellstudied tool in East Africa. Nevertheless, its performance in predicting outcomes among critically ill patients admitted to ICU's in Kenya (14) and Rwanda (15) are contradictory. External validation of SAPS 3 among patients admitted to the ICU's in Austria, Brazil and Italy reported that SAPS 3 had a good ability to predict outcomes but performed poorly across all probabilities of death when compared with APACHE-IV and $\mathrm{MPM}_{0}$-III (16-18). SAPS 3 may have greater potential for international use since the 
99

100

101

102

103

104

105

106

107

108

109

110

111

112

113 This was a single centre retrospective cohort study, conducted in the ICU of the Aga Khan Hospital, Dar-

114 es- Salaam, Tanzania. The Aga Khan Hospital is the largest private hospital and the only Joint

115 Commission International (JCI) accredited hospital in Tanzania. The ICU of the Aga Khan Hospital is a

11615 bed unit which provides level III services to all kind of critically ill patients. The ICU is capable of

117 providing mechanical ventilation, inotropic support and renal replacement therapy. The unit is divided in

1183 sections -7 adult general ICU beds (including 2 isolation rooms), 4 for cardiac patients and 4 for

119 paediatrics. The ICU is an open-model one run by a multidisciplinary team comprising of physician of the

120 primary specialty of care, physiotherapist and dietician led by a full-time critical care specialist. The

121

122

123

124

125

126

127

128

129

130

131

score was derived from data in more than one country (11). No study to date has assessed the performance of SAPS 3 in LMICs, especially in Sub-Saharan Africa.

These aforementioned predictive scoring systems have been compared in different studies and have produced variable results. The existence of a large number of scoring systems with contrasting performance suggests the best fit model is ICU specific. Thus, each particular ICU needs to determine which scoring system performs best in its setup; hence there was a need to carry out a comparative study in our cohort of patients to identify the best performing model. There have been no studies done in Tanzania that have compared the performance of these scoring models. The study had two main

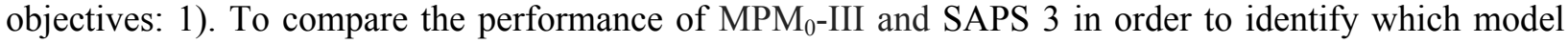
best fits in the ICU of the Aga Khan Hospital, Dar es Salaam. 2). To identify disease patterns and risk factors associated with higher mortality rates among critically ill patients. nurse-to-patient ratio ranges between 1:1 and 1:2. All adult patients aged 18 and above admitted to the ICU were eligible for the study. Patients admitted for observation, having incomplete data and those whose duration of stay in the unit was less than an hour as well as those diagnosed with COVID-19 were excluded from the study. Admissions to the ICU are limited to those meeting a strict admitting criteria set by the hospital. A total of 747 adults patients were admitted to the ICU from August 2018 to April 2020. A sample size of 331 patients having a specific outcome (death or discharge home) was determined to be sufficient to give the study a $80 \%$ power and $95 \%$ confidence for detection of $10 \%$ difference in performance between SAPS 3 and $\mathrm{MPM}_{0}$-III. The ICU admission register was used to identify patients admitted and patient files were retrieved from the medical records. The medical file numbers were entered into a computer and computer generated random sampling was performed until the desired sample size was achieved. Patient demographics and clinical data were extracted using patient records and were 
132 entered into a spreadsheet on Microsoft Office Excel 2010 (Redmond, WA, USA). Data was extracted by

133 experienced junior doctors with working experience in the ICU and was independently verified by the

134 primary author for accuracy and completeness. The reasons for admission were grouped into 11

135 categories: surgery, gastroenterology, neurology, endocrinology, respiratory, cardiovascular, nephrology,

136 sepsis, oncology, hematology, obstetrics and gynecology. When multiple diagnoses were present, the

137 leading one with the worst prognosis was selected as the main reason for admission.

138

139 Descriptive analysis of demographic characteristics were done and presented as percentages while the 140 categorical and continuous outcome variables were analysed and presented as means and medians with 141 interquartile ranges respectively. Categorical and continuous variables between survivors and non142 survivors were compared using Pearson's chi-square test and Mann-Whitney $U$ test respectively. SAPS-3 143 and $\mathrm{MPM}_{0}$-III were calculated using an online scoring calculator, available on www.uptodate.com. 144 Accurate discrimination and calibration are key distinguishing features that should be met by all 145 predictive scoring models. Discrimination of the model was assessed by the area under the receiver 146 operating characteristic (ROC) curve. An area of $0.7-0.8$ is reflected as fair, $0.8-0.9$ good and $>0.9$ 147 excellent. Non-parametric Wilcoxon statistics was used to compare the area under the ROC curves (19). 148 A Hosmer-Lemeshow goodness-of-fit test which follows chi-square distribution was used to evaluate the 149 model fit as well as calibration of the models with a p-value of $>0.05$ signifying no evidence of poor fit 150 (19). However, all other statistical tests with a p-value of $<0.05$ were considered statistically significant. 151 Any variable with P-Value $<0.05$ and those considered clinically significant in explaining mortality in the 152 ICU were considered in multivariable model. Determinants of mortality among critically ill patients were 153 identified using binary logistic regression; odds ratio with corresponding 95\% Confidence intervals (CI) 154 and P-value were reported. All statistical analysis was done using STATA version 15. The study protocol 155 was approved by the Ethical Research Committee (ERC) of the Aga Khan University (AKU/2020/051/fb) 156 and individual consent of each study participant was exempted since it did not affect the rights and 157 welfare of the patients.

158 RESULTS

\section{General characteristics of the participants}

160 A total of 331 patients were included in the study. Out of the 331 patients $(n=278,83.9 \%)$ survived and $161(\mathrm{n}=53,16.1 \%)$ died. Table 1 below shows general and clinical characteristic of the cohort and provides a 162 comparison of survivors to non-survivors. The median age of the cohort was 58 years (IQR 43-71) with 163 more than half of admitted patients being male $(n=208,62.8 \%)$ and of African origin $(n=178,53.8 \%)$..

164 Most of the patients were admitted to the ICU from the emergency department $(n=306,92.5 \%)$, who were 
165 at home prior $(\mathrm{n}=318,96.1 \%)$ with majority of them suffering from neurological disease $(\mathrm{n}=63,19 \%)$, 166 sepsis $(n=60,18.1 \%)$, respiratory $(n=36,10.9 \%)$ and cardiovascular $(n=36,10.9 \%)$ related conditions.

167 Median ICU and hospital LOS were 4 (2-6) and 6 (4-10) days respectively.

168

169 When survivors and non-survivors were compared, there was a statistically significant difference (P-value $170<0.05)$ in age, length of ICU stay, admitting category and code status. Higher mortality rates were noted 171 in the elderly aged between $75-84$ years $(n=15,28.3 \%)$, those suffering from sepsis $(n=13,24.5 \%)$ and 172 neurological diseases $(\mathrm{n}=10,18.9 \%)$. Higher percentage of mortality was also noted in Do-Not173 Resuscitate (DNR) patients $(n=27,50.9 \%)$ compared to those without limitations of care. There was no 174 statistically significant difference between survivors and non-survivors by sex, ethnicity and prior 175 location before ICU admission. The overall SAPS 3 score for all the patients was 42 (IQR: 32-51) of 176 which non-survivors had a higher score 60 (IQR: 51-68) than the survivors 39 (IQR: 31-48) with p-value $177(<0.0001)$. Similarly, the median $\mathrm{MPM}_{0}$-III score in non-survivors, 5 (IQR: 4-6) was higher than 178 survivors, 3 (IQR: 2-4) with p-value $(<0.0001)$.

Table 2 below, illustrates the type of support patients received in the first 24 hours of ICU admission. Of the 331 patients admitted to the ICU, $(n=123,37.2 \%)$ patients received support in the first hour of ICU admission that included: mechanical ventilation, inotropes and hemodialysis. Highest mortality was noted amongst those who required inotropic support and mechanical ventilation $(n=21,39.6 \%)$ in the first hour of ICU admission.

Table 3 below highlights comorbid conditions amongst the critical ill patients admitted to the ICU. More than one comorbid condition per critically ill patient was recorded when present. The most common comorbid condition amongst our cohort was hypertension $(\mathrm{n}=174,52.6 \%)$ and diabetes mellitus $(\mathrm{n}=107$, $32.3 \%$ ). When comorbid conditions were compared between survivors and non- survivors, a higher percentage of mortality with statistically significant difference ( $\mathrm{P}$ value $<0.05$ ) was noted among those suffering with chronic kidney disease $(n=14,26.4 \%)$ and liver cirrhosis $(n=7,13.2 \%)$.

Calibration of each scoring system exhibited good performance. The goodness of fit Hosmer-Lemeshow test and p-value of each scoring system is shown in Table 4 below. The area under ROC of SAPS 3 and $\mathrm{MPM}_{0}$-III in prediction of mortality are shown below in Figure 1 below. The area under the ROC was calculated to evaluate the predictive value of the scoring systems. The area under the ROC curve for the 
199 SAPS 3 showed a statistically significant predictive marker of mortality (AUC: 0.8892; 95\%CI: 0.844-

200 0.935). The cut-off value for SAPS 3 was 54 with the sensitivity of $72 \%$ and specificity of $90 \%$. The

$201 \mathrm{MPM}_{0}$-III scoring system also showed a statistically significant predictive marker for the outcome of

202 interest (AUC: 0.904; 95\%CI: 0.864-0.944). The cut-off value for $\mathrm{MPM}_{0}$-III was 4, with sensitivity of

$20374 \%$ and specificity of $87 \%$. There was no statistically significant difference between the ROC curves of

204 the two models (P-value $=0.2418)$ (Table 5).

205

206

207

208

209

210

211

212

213

214

215

216

217

218

219

220

221

222

223

224

225

226

227

228

229

230

231

232

233

The overall estimated median (IQR) predicted mortality among the 331 ICU patients was $6 \%$ (2\%-20\%) on the SAPS 3 model and $11.5 \%(3.8 \%-27.9 \%)$ based on the $\mathrm{MPM}_{0}$-III model. The stratified analysis by survivors and non-survivors is shown in Figure 2 below. The median predicted mortality risk for survivors is lower than those of non-survivors. In the SAPS 3 model, the estimated median for survivors was $5 \%$ (IQR: $1 \%-11 \%$ ) while for the non-survivors this was $50 \%$ (IQR: $34 \%-69 \%$ ) Based on the $\mathrm{MPM}_{0}$-III model the median predicted mortality was $9.1 \%$ (3.1\%-1.7\%) and $68.5 \%$ (IQR: $42.7 \%-84.0 \%$ ) for survivors and non-survivors respectively.

Multiple clinical factors were associated with increased adjusted odds of mortality. These included length of ICU stay (adjusted odds ratio [aOR], 1.462; P-value $=0.001$ ) and those transferred from the ward (aOR, 5.341; P -value $<0.022$ ). However, it was protective to stay longer in the hospital as the odds of mortality decreased as the length of hospitalization increased (aOR, 0.717; P-value $=0.002)$ (Table 6).

\section{DISCUSSION}

To our knowledge, this is the first study to report on performance of predictive scoring models in Tanzania and more so in a private setting. Accurate discrimination and calibration are two key characteristics that should be met by all predictive scoring systems. Both SAPS 3 and MPM $\mathrm{M}_{0}$-III performed well in our cohort. According to our results, a SAPS 3 score of higher than 54 can predict mortality with sensitivity of $72 \%$ and specificity of $90 \%$. A $\mathrm{MPM}_{0}$-III score of greater than 4 can predict mortality with sensitivity of $74 \%$ and specificity of $87 \%$.

Discrimination describes the accuracy of a given prediction. In our cohort, the discriminatory capability of both SAPS 3 (20 variables) and $\mathrm{MPM}_{0}$-III (16 variables) was good. There was no statistically 
234 significance difference when both these models were compared, suggesting that the model with more

235 variables was not associated with better discriminatory performance. MPM $_{0}$-III has been externally 236 validated in various ICUs in North America $(12,13,20)$ and has shown to have good discrimination 237 which was similar to our study finding. However, a study done at Aga Khan University Hospital, 238 Nairobi, Kenya (14) and two public ICUs in Rwanda(15) showed MPM $_{0}$-III to have fair discrimination 239 amongst their cohort. This observed difference in discrimination may be due to the effect of differences in 240 proportion of case mixes between the study settings. Similarly SAPS 3 has been externally validated in 241 various ICUs in Italy (16), Brazil (17), Austria (18) and found to have good discriminatory capability 242 amongst their cohort. Despite SAPS 3 having greater prospective for international generalizability there 243 has been no published studies evaluating its performance in Sub-Saharan African ICUs. This is the first 244 study that reports its potential for application in LMICs.

Calibration describes how the instrument performs over a wide range of predicted mortalities. Calibration is sensitive to alterations in case-mix and patient care and interventions. Despite its tendency to

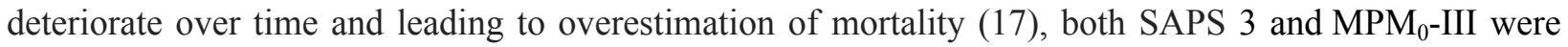
well calibrated amongst the critically ill patients admitted at our study setting. Our study findings were contrary to SAPS 3 validation studies mentioned earlier which reported poor calibration and overestimation of mortality (16-18). However, external validation studies have reported $\mathrm{MPM}_{0}$-III to have good calibration $(12,13,20)$. Earlier studies mentioned that were conducted in Sub-Saharan Africa have produced contrasting results. The $\mathrm{MPM}_{0}$-III was well calibrated amongst the critical ill patients admitted to the ICU of the Aga Khan University Hospital, Nairobi (14) but showed poor calibration amongst all adult patients admitted to Rwanda's two public ICUs (15). These findings highlight the similar treatment protocols and interventions between two sister hospitals located in different geographical regions.

In this retrospective study we also aimed to identify patient demographics, disease patterns, clinical outcomes as well as factors associated with higher risk of mortality in patients admitted to the ICU of the Aga Khan Hospital, Dar -es Salaam. Based on this retrospective observational cohort, the in-hospital mortality of critically ill patients was $16.1 \%$, which is far less than the reported mortality among all other tertiary referral hospitals in Tanzania, 41.4\% (21) but slightly exceeds rates reported in western Europe and North America (1). This disparity is not surprising since the intensive care unit at our setting has access to more resources than similar units in the country and comparable in various ways to facilities in HICs. The ICU cohort studied in the four tertiary referral hospitals in Tanzania was younger (median age 34 years, IQR 21-53) compared to our study population (median age 58 years, IQR 43-71). This variation 
268

269

270

271

272

273

274

275

276

277

278

279

280

281

282

283

284

285

286

287

288

289

290

291

292

293

294

295

296

297

298

299

300

301

could be due to the exclusion of patients aged less than 18 years in our study. However both the cohorts had male predominance of $57.5 \%$ and $62.8 \%$ respectively (21). The bulk of admissions in our cohort were those suffering from neurological disease, sepsis, respiratory and cardiovascular related conditions. Mortality was highest among those admitted due to sepsis. Our results are in parallel with a large intercontinental database that emphasized the association of sepsis with high mortality rates in all countries (1).The median length of ICU stay is similar to reports from tertiary hospitals in Sub-Saharan Africa $(21,22)$.

Prolonged length of stay (LoS) in the ICU and patients transferred from the general ward to the ICU were factors associated with higher adjusted odds of mortality among critically ill patients. Prolonged LoS in the ICU may be attributed to development of multi- systemic complications necessitating continued organ support. There are no laws and guidelines in Tanzania with regards to withdrawal of support, hence we hypothesize that significant fraction of patients with a prolonged course of illness and with expected poor outcomes are admitted for extended intervals before succumbing to death. Our study findings are comparable to several studies done in well-equipped ICUs that concluded patients with multiple diseases and having organ dysfunction were key factors that prolong the $\operatorname{LoS}$ in $\operatorname{ICU}(23,24)$. Contrasting results have also been published that LoS in ICU is not an independent risk factor for inhospital mortality (25). Those patients transferred from the general ward to the ICU also had higher adjusted odds of mortality; this is not surprising since it is a mere reflection of deteriorating physiological and clinical condition. Few studies have demonstrated early transfer to the ICU for treatment to have a substantial impact on in-hospital mortality and $\operatorname{LoS}(26,27)$.

We identified several limitations in our study. Firstly, this was a single center study and as such the findings may not be valid across all patient populations in Tanzania. Secondly, since our study was a retrospective design it restricted us the ability to follow up outcomes after ICU discharge and doesn't provide the same level of evidence as a prospective study design.

\section{CONCLUSION}

In summary, this is the first and largest study to report on performance of predictive scoring models in Tanzania. Our study concluded both SAPS 3 and MPM $_{0}$-III perform well among critically ill patients admitted to the ICU of the Aga Khan Hospital, Dar es Salaam, Tanzania. We found our mortality among critically ill patients to be much lower compared to other tertiary referral hospitals in Tanzania. Amongst out cohort, patients with sepsis had the highest mortality rate. Thus clinical research targeting infection prevention efforts and early implementation of targeted interventions would be important to improving 
302 outcomes. Prolonged ICU stay and transfer from general wards to ICU were key factors of mortality. Of

303 note, the performance of predictive scoring models tend to deteriorate over time; termed as worsening of

304 discrimination and calibration and resulting in overestimation of mortality (17). Thus periodic updating is

305 crucial for sustaining accuracy of these predictive models. .

306 ABBREVIATIONS

307 - APACHE: Acute physiology and chronic health evaluation

308 - SAPS: Simplified acute physiology score

309 - MPM: Mortality probability models

310 - LoS : Length of Stay

311 - ICU :- Intensive care Unit

\section{DECLARATION}

313

314 Ethics approval and consent to participate

315 The study was approved by the Aga Khan University Ethical Research Committee (AKU-ERC, EA). The

316 National Institute for Medical Research (NIMR) mandates the AKU-ERC to approve health research

317 conducted by Tanzanian staff and students under the Act of Parliament No. 23 of 1979 and its

318 amendments in 1997.

319 Consent for publication

320 Not Applicable

321

322

323

324

325

326

327

328

329

330

331

332

333

\section{Acknowledgements}

Special thanks to Dr. Kamran Hameed, head of the Internal medicine department at the Aga Khan University and hospital, Dar-es-Salaam for his continuous mentorship and guidance during my residency program.

\section{Author Details}

1. Department of Internal Medicine, Aga Khan University, Medical College, East Africa

2. Department of Family Medicine, Aga Khan University, Medical College, East Africa

3. Department of Internal Medicine, Aga Khan Hospital, Dar-es-Salaam, Tanzania

4. Department of Population Health, Aga Khan University, Medical College, East Africa

5. Department of Medicine \& Pharmacy, Texas A\&M University, Texas, USA 
335 REFERENCE

336 1. Vincent JL, Marshall JC, Namendys-Silva SA, Francois B, Martin-Loeches I, Lipman J, et al.

337 Assessment of the worldwide burden of critical illness: the intensive care over nations (ICON) audit.

338 Lancet Respir Med. 2014;2(5):380-6.

$3392 . \quad$ Ilori IU, Kalu QN. Intensive care admissions and outcome at the University of Calabar Teaching

340 Hospital, Nigeria. J Crit Care. 2012;27(1):105 e1-4.

3413 3. Smith ZA, Ayele Y, McDonald P. Outcomes in critical care delivery at Jimma University

342 Specialised Hospital, Ethiopia. Anaesth Intensive Care. 2013;41(3):363-8.

343 4. Baker T, Lugazia E, Eriksen J, Mwafongo V, Irestedt L, Konrad D. Emergency and critical care

344 services in Tanzania: a survey of ten hospitals. BMC Health Serv Res. 2013;13:140.

345 5. Dondorp AM, lyer SS, Schultz MJ. Critical Care in Resource-Restricted Settings. JAMA.

346 2016;315(8):753-4.

347 6. Adhikari NK, Fowler RA, Bhagwanjee S, Rubenfeld GD. Critical care and the global burden of

348 critical illness in adults. Lancet. 2010;376(9749):1339-46.

$3497 . \quad K e e g a n$ MT, Gajic O, Afessa B. Severity of illness scoring systems in the intensive care unit. Crit 350 Care Med. 2011;39(1):163-9.

$351 \quad 8 . \quad$ Zimmerman JE, Wagner DP, Knaus WA, Williams JF, Kolakowski D, Draper EA. The use of risk

352 predictions to identify candidates for intermediate care units. Implications for intensive care utilization

353 and cost. Chest. 1995;108(2):490-9.

$3549 . \quad J u n e j a ~ D$, Singh O, Nasa P, Dang R. Comparison of newer scoring systems with the conventional

355 scoring systems in general intensive care population. Minerva Anestesiol. 2012;78(2):194-200.

356 10. Zimmerman JE, Kramer AA, McNair DS, Malila FM. Acute Physiology and Chronic Health

357 Evaluation (APACHE) IV: hospital mortality assessment for today's critically ill patients. Crit Care Med.

358 2006;34(5):1297-310.

359 11. Metnitz PG, Moreno RP, Almeida E, Jordan B, Bauer P, Campos RA, et al. SAPS 3--From

360 evaluation of the patient to evaluation of the intensive care unit. Part 1: Objectives, methods and cohort

361 description. Intensive Care Med. 2005;31(10):1336-44.

362 12. Higgins TL, Teres D, Copes WS, Nathanson BH, Stark M, Kramer AA. Assessing contemporary

363 intensive care unit outcome: an updated Mortality Probability Admission Model (MPMO-III). Crit Care

364 Med. 2007;35(3):827-35.

365 13. Kuzniewicz MW, Vasilevskis EE, Lane R, Dean ML, Trivedi NG, Rennie DJ, et al. Variation in ICU

366 risk-adjusted mortality: impact of methods of assessment and potential confounders. Chest.

367 2008;133(6):1319-27.

368 14. Lukoko LN, Kussin PS, Adam RD, Orwa J, Waweru-Siika W. Investigating SOFA, delta-SOFA and

369 MPM-III for mortality prediction among critically ill patients at a private tertiary hospital ICU in Kenya: A

370 retrospective cohort study. PLoS One. 2020;15(7):e0235809.

371 15. Riviello ED, Kiviri W, Fowler RA, Mueller A, Novack V, Banner-Goodspeed VM, et al. Predicting

372 Mortality in Low-Income Country ICUs: The Rwanda Mortality Probability Model (R-MPM). PLoS One.

373 2016;11(5):e0155858.

374 16. Poole D, Rossi C, Anghileri A, Giardino M, Latronico N, Radrizzani D, et al. External validation of

375 the Simplified Acute Physiology Score (SAPS) 3 in a cohort of 28,357 patients from 147 Italian intensive

376 care units. Intensive Care Med. 2009;35(11):1916-24.

377 17. Nassar AP, Jr., Mocelin AO, Nunes AL, Giannini FP, Brauer L, Andrade FM, et al. Caution when

378 using prognostic models: a prospective comparison of 3 recent prognostic models. J Crit Care.

379 2012;27(4):423 e1-7.

380 18. Metnitz B, Schaden E, Moreno R, Le Gall JR, Bauer P, Metnitz PG, et al. Austrian validation and

381 customization of the SAPS 3 Admission Score. Intensive Care Med. 2009;35(4):616-22. 
382 19. Steyerberg EW, Vickers AJ, Cook NR, Gerds T, Gonen M, Obuchowski N, et al. Assessing the 383 performance of prediction models: a framework for traditional and novel measures. Epidemiology. 384 2010;21(1):128-38.

385 20. Higgins TL, Kramer AA, Nathanson BH, Copes W, Stark M, Teres D. Prospective validation of the 386 intensive care unit admission Mortality Probability Model (MPMO-III). Crit Care Med. 2009;37(5):1619-

38723.

388 21. Sawe HR, Mfinanga JA, Lidenge SJ, Mpondo BC, Msangi S, Lugazia E, et al. Disease patterns and 389 clinical outcomes of patients admitted in intensive care units of tertiary referral hospitals of Tanzania.

390 BMC Int Health Hum Rights. 2014;14:26.

391 22. Kwizera A, Dunser M, Nakibuuka J. National intensive care unit bed capacity and ICU patient 392 characteristics in a low income country. BMC Res Notes. 2012;5:475.

393 23. Toptas M, Sengul Samanci N, Akkoc I, Yucetas E, Cebeci E, Sen O, et al. Factors Affecting the 394 Length of Stay in the Intensive Care Unit: Our Clinical Experience. Biomed Res Int. 2018;2018:9438046.

395 24. Moitra VK, Guerra C, Linde-Zwirble WT, Wunsch H. Relationship Between ICU Length of Stay and 396 Long-Term Mortality for Elderly ICU Survivors. Crit Care Med. 2016;44(4):655-62.

397 25. Williams TA, Ho KM, Dobb GJ, Finn JC, Knuiman M, Webb SA, et al. Effect of length of stay in 398 intensive care unit on hospital and long-term mortality of critically ill adult patients. Br J Anaesth. 399 2010;104(4):459-64.

400 26. Churpek MM, Wendlandt B, Zadravecz FJ, Adhikari R, Winslow C, Edelson DP. Association 401 between intensive care unit transfer delay and hospital mortality: A multicenter investigation. J Hosp 402 Med. 2016;11(11):757-62.

403 27. Young MP, Gooder VJ, McBride K, James B, Fisher ES. Inpatient transfers to the intensive care 404 unit: delays are associated with increased mortality and morbidity. J Gen Intern Med. 2003;18(2):77-83.

405

406

407

408

409 
Figure 1

Receiver operating curve for predicting mortality according to SAPS 3 and MPM III models

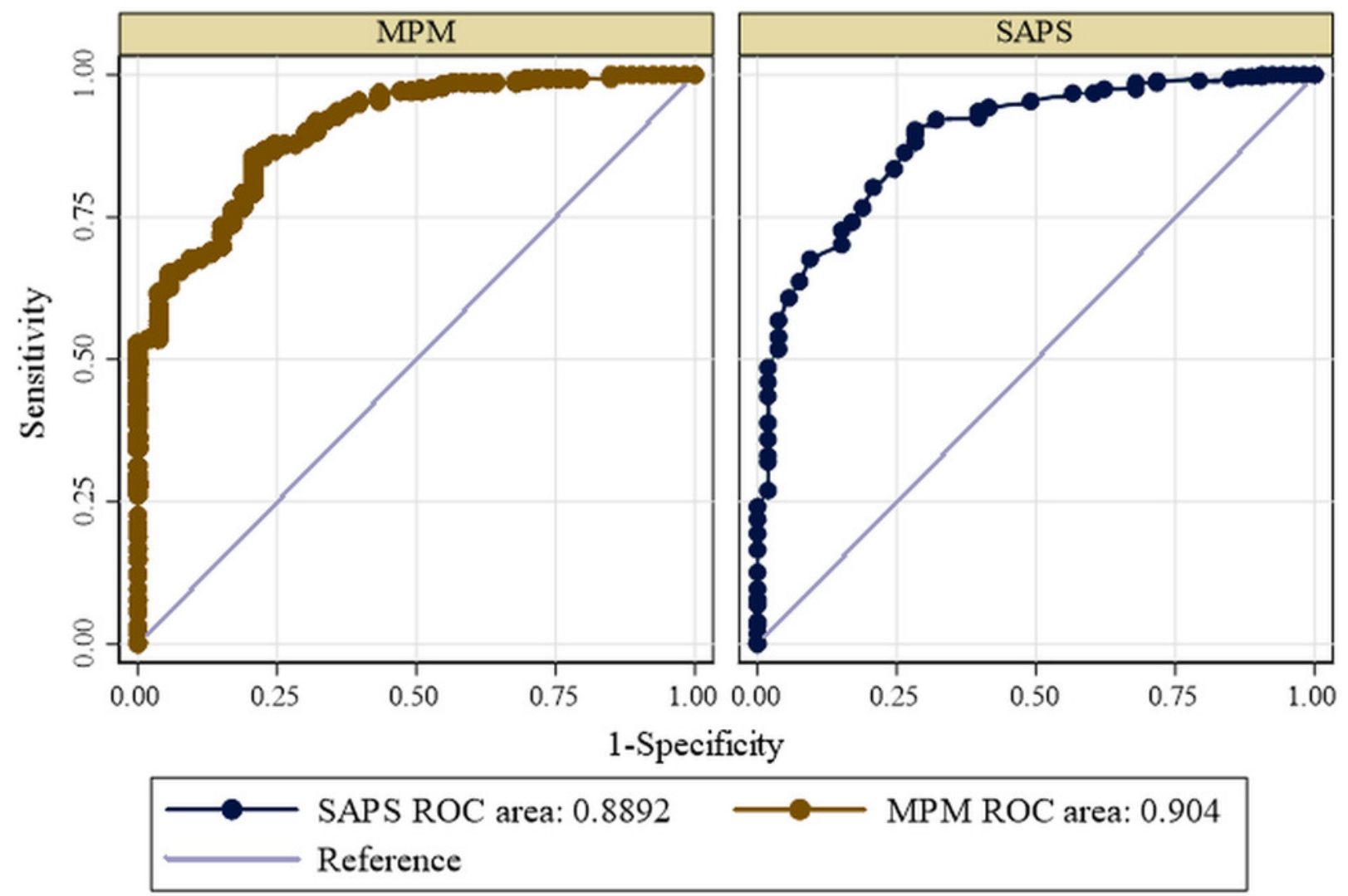


Figure 2

Median predicted mortality rates for SAPS 3 and $\mathrm{MPM}_{0}-\mathrm{III}$

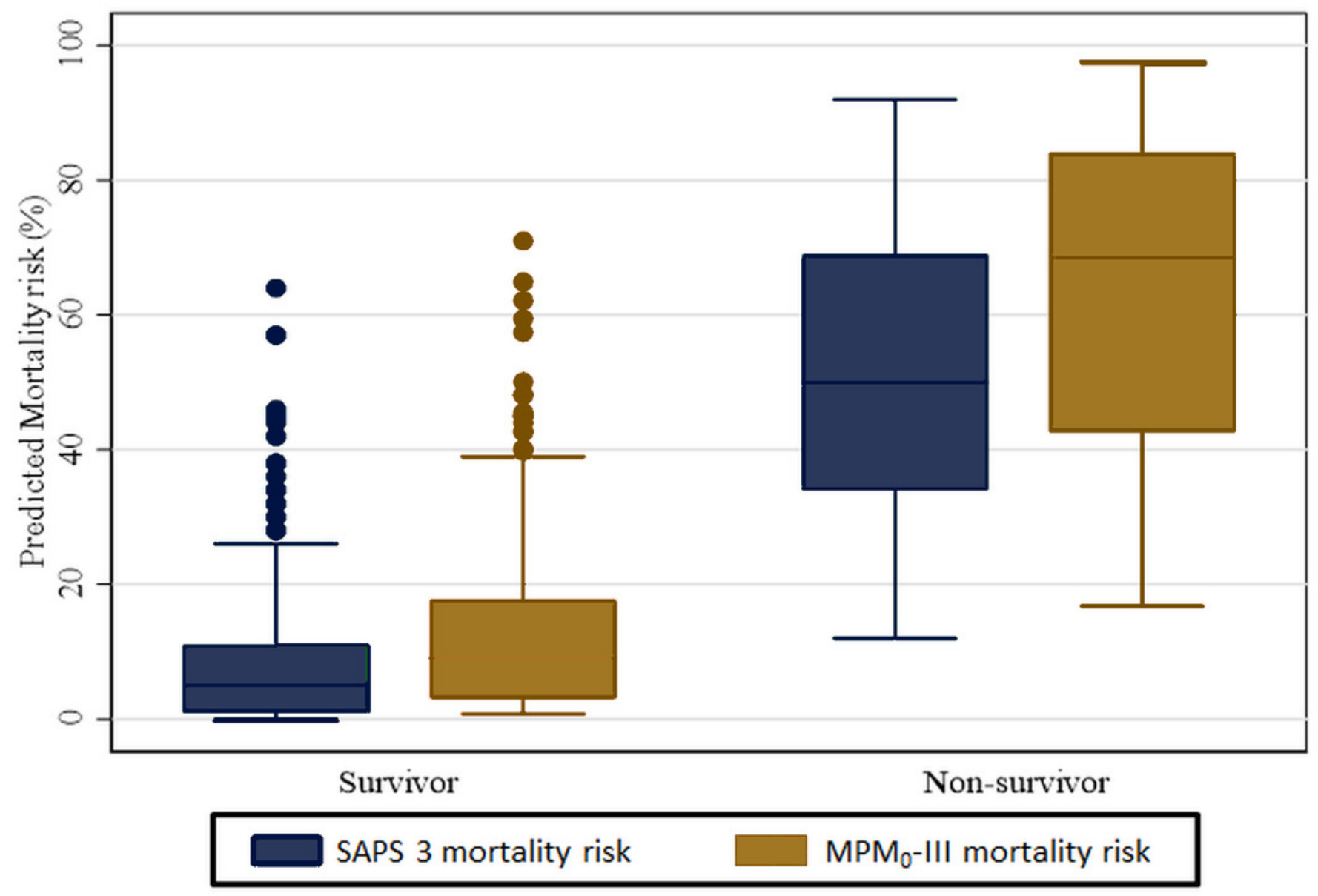




\section{Table 1 (on next page)}

General and clinical characteristics of patients $(\mathrm{N}=331)$ 
1 Table 1: General and clinical characteristics of patients $(\mathbf{N}=331)$

\begin{tabular}{|c|c|c|c|c|}
\hline \multirow{2}{*}{ Characteristics } & $\operatorname{All}(\mathrm{N}=331)$ & Survivors $(\mathrm{n}=278)$ & Non-survivors $(\mathrm{n}=53)$ & \multirow{2}{*}{ p-value } \\
\hline & $\mathrm{N}(\%)$ & $\mathrm{n}(\%)$ & $\mathrm{n}(\%)$ & \\
\hline \multicolumn{5}{|l|}{ Sex } \\
\hline Male & $208(62.8)$ & $174(62.6)$ & $34(64.2)$ & \multirow[t]{2}{*}{0.829} \\
\hline Female & $123(37.2)$ & $104(37.4)$ & $19(35.8)$ & \\
\hline \multicolumn{5}{|l|}{ Age group in years } \\
\hline$<45$ & $87(26.3)$ & $79(28.4)$ & $8(15.1)$ & \multirow{5}{*}{0.015} \\
\hline $45-64$ & $114(34.4)$ & $99(35.6)$ & $15(28.3)$ & \\
\hline $65-74$ & $61(18.4)$ & $51(18.4)$ & $10(18.9)$ & \\
\hline $75-84$ & $51(15.4)$ & $36(12.9)$ & $15(28.3)$ & \\
\hline$>84$ & $18(5.4)$ & $13(4.7)$ & $5(9.4)$ & \\
\hline \multicolumn{5}{|l|}{ Ethnicity } \\
\hline African & $178(53.8)$ & $152(54.7)$ & $26(49.1)$ & \multirow{3}{*}{0.589} \\
\hline Asian & $136(41.1)$ & $111(39.9)$ & $25(47.2)$ & \\
\hline Other & $17(5.1)$ & $15(5.4)$ & $2(3.8)$ & \\
\hline \multicolumn{5}{|l|}{ Admitted from } \\
\hline Emergency & $306(92.5)$ & $257(92.5)$ & $49(92.5)$ & \multirow{3}{*}{0.800} \\
\hline Wards & $15(4.5)$ & $12(4.3)$ & $3(5.7)$ & \\
\hline Clinic & $10(3.0)$ & $9(3.2)$ & $1(1.9)$ & \\
\hline \multicolumn{5}{|c|}{ Location before ICU admission } \\
\hline Home & $318(96.1)$ & $267(96.0)$ & $51(96.2)$ & \multirow[t]{2}{*}{0.950} \\
\hline Hospital & $13(3.9)$ & $11(4.0)$ & $2(3.8)$ & \\
\hline \multicolumn{4}{|l|}{ Admitting Category } & \multirow[t]{12}{*}{0.014} \\
\hline Surgery & $38(11.5)$ & $36(12.9)$ & $2(3.8)$ & \\
\hline Gastroenterology & $31(9.4)$ & $25(9.0)$ & $6(11.3)$ & \\
\hline Neurology & $63(19.0)$ & $53(19.1)$ & $10(18.9)$ & \\
\hline Endocrinology & $18(5.4)$ & $17(6.1)$ & $1(1.9)$ & \\
\hline Respiratory & $36(10.9)$ & $35(12.6)$ & $1(1.9)$ & \\
\hline Cardiovascular & $36(10.9)$ & $30(10.8)$ & $6(11.3)$ & \\
\hline Nephrology & $16(4.8)$ & $12(4.3)$ & $4(7.6)$ & \\
\hline Sepsis & $60(18.1)$ & $47(16.9)$ & $13(24.5)$ & \\
\hline Obstetrics and Gynecology & $10(2.0)$ & $9(3.2)$ & $1(1.9)$ & \\
\hline Hematology & $7(2.1)$ & $5(71.4)$ & $2(3.8)$ & \\
\hline Oncology & $16(4.8)$ & $9(3.2)$ & $7(13.2)$ & \\
\hline \multicolumn{5}{|l|}{ Code Status on Admission } \\
\hline DNR & $40(12.1)$ & $13(4.7)$ & $27(50.9)$ & \multirow[t]{2}{*}{$<0.001$} \\
\hline Full Code & $291(87.9)$ & $265(95.3)$ & $26(49.1)$ & \\
\hline Age in years & $58(43-71) \dagger$ & $55.5(41-70) \dagger$ & $70(55-78) \dagger$ & $0.0003 \ddagger$ \\
\hline SAPS 3 scores & $42(32-51) \dagger$ & $39(31-48) \dagger$ & $60(51-68) \dagger$ & $<0.0001 \uparrow$ \\
\hline MPM $_{0}$ III scores & $3(2-4) \dagger$ & $3(2-4) \dagger$ & $5(4-6) \dagger$ & $<0.0001 t$ \\
\hline LOS ICU (days) & $4(2-6) \dagger$ & $4(2-6) \dagger$ & $6(2-11) \dagger$ & $0.0029 \ddagger$ \\
\hline LOS Hospital (days) & $6(4-10) \dagger$ & $6(4-10) \dagger$ & $8(3-13) \dagger$ & $0.3248 \div$ \\
\hline
\end{tabular}

2

3 †median (IQR); †: p-value for Mann-Whitney U test; LOS: Length of Stay, DNR: Do Not

4 resuscitate. Data in median (IQR), and $n(\%)$ 
Table 2 (on next page)

Type of support received in the first hour of ICU admission 
1 Table 2: Type of support received in the first hour of ICU admission

\begin{tabular}{lcccc}
\hline \multirow{2}{*}{ Type of support } & $\mathrm{All}(\mathrm{N}=331)$ & Survivors $(\mathrm{n}=278)$ & Non-survivors $(\mathrm{n}=53)$ & $\mathrm{p}$-value \\
\cline { 2 - 4 } & $\mathrm{N}(\%)$ & $\mathrm{n}(\%)$ & $\mathrm{n}(\%)$ & \\
\hline None & $208(62.8)$ & $196(70.5)$ & $12(22.6)$ & $<0.001$ \\
Hemodialysis & $13(3.9)$ & $11(4.0)$ & $2(3.8)$ & 0.9498 \\
Inotropes & $33(10.0)$ & $29(10.4)$ & $4(7.6)$ & 0.5206 \\
Mechanical ventilation & $26(7.9)$ & $17(6.1)$ & $9(17.0)$ & 0.0070 \\
Inotropes, Hemodialysis & $5(1.5)$ & $5(1.8)$ & $0(0.0)$ & 0.3252 \\
Inotropes, mechanical ventilation & $36(10.9)$ & $15(5.4)$ & $21(39.6)$ & $<0.001$ \\
Mechanical ventilation, & $3(0.9)$ & $3(1.1)$ & $0(0.0)$ & 0.4474 \\
Hemodialysis & & & & \\
Inotropes, mechanical ventilation, & $7(2.1)$ & $2(0.7)$ & $5(9.4)$ & 0.0001 \\
hemodialysis & & & &
\end{tabular}

2

3 Data presented in $n(\%)$ 
Table 3 (on next page)

Comorbid conditions among critically ill patients admitted to the ICU 
1 Table 3: Comorbid conditions among critically ill patients admitted to the ICU

2

\begin{tabular}{lcccc}
\hline \multirow{2}{*}{ Comorbidity } & All $(\mathrm{N}=331)$ & Survivors $(\mathrm{n}=278)$ & Non-survivors $(\mathrm{n}=53)$ & \\
\cline { 2 - 4 } & $\mathrm{N}(\%)$ & $\mathrm{n}(\%)$ & $\mathrm{n}(\%)$ & \\
\hline Hypertension & $174(52.6)$ & $146(52.5)$ & $28(52.8)$ & 0.9553 \\
Diabetes Mellitus & $107(32.3)$ & $87(31.3)$ & $20(37.7)$ & 0.3613 \\
Heart Failure & $53(16.0)$ & $41(14.7)$ & $12(22.6)$ & 0.1510 \\
Chronic Kidney Disease & $45(13.6)$ & $31(11.2)$ & $14(26.4)$ & 0.0030 \\
HIV & $21(6.3)$ & $16(5.8)$ & $5(9.4)$ & 0.314 \\
COPD & $17(5.1)$ & $15(5.4)$ & $2(3.8)$ & 0.6239 \\
CAD & $17(5.1)$ & $15(5.4)$ & $2(3.8)$ & 0.6239 \\
Liver Cirrhosis & $16(4.8)$ & $9(3.2)$ & $7(13.2)$ & 0.0022 \\
\hline & & & & \\
DM \& HTN & $87(26.3)$ & $72(25.9)$ & $15(28.3)$ & 0.7157 \\
HTN \& CKD & $39(11.8)$ & $27(9.7)$ & $12(22.6)$ & 0.0075 \\
DM \& HTN \& CKD & $30(9.1)$ & $21(7.6)$ & $9(17.0)$ & 0.0285 \\
\hline
\end{tabular}

3 Data presented in $n(\%)$

4 
Table 4 (on next page)

Goodness of fit Hosmer-Lemeshow test and p-value of each scoring model 
1 Table 4: Goodness of fit Hosmer-Lemeshow test and p-value of each scoring model 2

\begin{tabular}{lcc}
\hline Scoring Model & Chi - Square & P - Value \\
\hline MPM 0- III Score & 5.08 & 0.2791 \\
SAPS 3 & 4.61 & 0.7980 \\
\hline
\end{tabular}




\section{Table 5 (on next page)}

Area under curve and 95\% confidence Intervals for the models 
1 Table 5: Area under curve and $95 \%$ confidence Intervals for the models

2

3

\begin{tabular}{lccccc}
\hline Variable & Cut-off & AUC & LL & UL & P-value \\
\hline MPM 0- III Score & 4 & 0.904 & 0.864 & 0.944 & 0.2418 \\
SAPS 3 & 54 & 0.8892 & 0.8440 & 0.935 & \\
\hline
\end{tabular}

4

5 LL: Lower limits; UL: Upper limits; AUC: Area under the ROC curve 6 
Table 6(on next page)

Factors associated with increased odds of mortality among critically ill patients 
1 Table 6: Factors associated with increased odds of mortality among critically ill patients

\begin{tabular}{lcccccc}
\hline \multirow{2}{*}{ Characteristics } & \multicolumn{3}{c}{ Unadjusted Odds ratio } & \multicolumn{3}{c}{ Adjusted Odds ratio } \\
\cline { 2 - 6 } & OR & $95 \%$ CI & P-value & OR & $95 \%$ CI & P-value \\
\hline Age in years & 1.032 & $1.014-1.051$ & 0.001 & 1.020 & $0.997-1.043$ & 0.086 \\
LOS in ICU & 1.068 & $1.021-1.117$ & $<0.001$ & 1.462 & $1.179-1.814$ & 0.001 \\
LOS in hospital & 1.017 & $0.994-1.041$ & 0.141 & 0.717 & $0.580-0.886$ & 0.002 \\
Sex & & & & & & \\
$\quad$ Male & ref & & & & & \\
$\quad$ Female & 0.935 & $0.507-1.723$ & 0.829 & 0.870 & $0.399-1.893$ & 0.725 \\
$\quad$ Admitted From & & & & & & \\
$\quad$ Emergency & ref & & & & & \\
$\quad$ Wards & 1.311 & $0.357-4.819$ & 0.683 & 5.341 & $1.278-22.322$ & 0.022 \\
$\quad$ Clinic & 0.583 & $0.072-4.704$ & 0.612 & 1.033 & $0.114-9.347$ & 0.977 \\
Code status & & & & & & \\
$\quad$ DNR & ref & & & & & \\
$\quad$ Full code & 0.047 & $0.022-0.102$ & $<0.001$ & 0.052 & $0.021-0.129$ & $<0.001$ \\
\hline
\end{tabular}

